\title{
Canine Transmissible Venereal Tumor
}

National Cancer Institute

\section{Source}

National Cancer Institute. Canine Transmissible Venereal Tumor. NCI Thesaurus. Code C162473.

A sexually transmitted tumor arising from histiocytes and occurring in dogs. 